\title{
Applicability of laser scanning cytometry to study paediatric alveolar macrophages
}

\author{
H.J. Bunn*, G. Woltmann*, J. Grigg*
}

\begin{abstract}
Applicability of laser scanning cytometry to study paediatric alveolar macrophages. H.J. Bunn, G. Woltmann, J. Grigg. (C) ERS Journals Ltd 2002.

ABSTRACT: Laser scanning cytometry (LSC) generates quantitative information on immune receptor expression from cells cytocentrifuged onto a microscope slide. In children, the description of developmental changes in immune receptor expression on alveolar macrophages (AM) has been limited by the small number of cells recovered by bronchoalveolar lavage (BAL). The applicability of LSC to the study of AM from normal children was therefore assessed.

AM were obtained by BAL of normal children following intubation prior to elective surgery. The ability of LSC to identify the cytoplasm of AM was assessed using either: 1) autofluorescence; 2) forward scatter; 3 ) nuclear staining with propidium iodide; or 4) a fluorescent-labelled monoclonal antibody to CD68, a pan-macrophage antigen.

LSC could only reliably identify individual AM when stained with CD68. The sensitivity for detecting single whole AM using CD68 was 0.97 and the positive predictive value was 0.88 , respectively, with excellent repeatability. In addition, a range of immunofluorescence parameters were generated for CD68.

It is concluded that laser scanning cytometry is suited to the study of immune receptor expression from small numbers of paediatric alveolar macrophages, when CD68 is used for cell identification.

Eur Respir J 2002; 20: 1437-1443.
\end{abstract}

*Dept of Child Health and ${ }^{*}$ Division of Respiratory Medicine, Glenfield Hospital, Institute for Lung Health, University of Leicester, UK.

Correspondence: J. Grigg, Senior Lecturer in Paediatric Respiratory Medicine, Leicester Children's Asthma Centre, University of Leicester, Leicester Royal Infirmary, LE2 7LX

Fax: 441162523282

E-mail: jg33@le.ac.uk

Keywords: Alveolar macrophage, bronchoalveolar lavage, child, laser scanning cytometry

Received: April 252002

Accepted after revision: July 162002

J. Grigg was supported by Medisearch and Henry Smith's Charity.
The measurement of developmental changes in alveolar macrophage (AM) immune receptor expression using flow cytometry (FC) in children is limited by several factors. First, the recommended weight-adjusted bronchoalveolar lavage (BAL) volume $[1,2]$ recovers only a small total number of AM. Secondly, normal healthy children must be recruited serendipitously prior to elective surgery, and cells must be stored for several weeks or months before batch processing. These are important deficiencies. For example, the authors have found that AM from young children are less suppressive for T-lymphocyte proliferation using in vitro functional assays [2], but the developmental changes of relevant immune receptors could not be identified because of the limited numbers of cells recovered. Animal models demonstrate changes in AM immune receptor expression during the process of normal development [3,4] but the relevance to humans has not been validated.

In contrast, the quantitative assessment of the immune receptor expression of human adult $\mathrm{AM}$ is less problematic, since large volumes of saline are instilled during BAL and the numbers of AM recovered and morphology are suitable for FC analysis [5]. Using $\mathrm{FC}$, fluorescent antibodies have attached to immune receptors on cells which are then identified as AM by their characteristic autofluorescence, light scatter properties (e.g. forward scatter) or a combination of both [6]. There are, however, limitations to FC; it cannot display the staining morphology without additional cell sorting, AM cannot be restained and must be processed either when viable or when freshly fixed. Furthermore, relatively large numbers are required to overcome significant processing losses [7]. To date, where AM numbers are limited or if AM morphology is required, image analysis (IA) systems such as confocal microscopy have been used [8]. IA is better suited to small numbers of cells and samples can be collected, stored and then stained as a batch [9]. However, data collection by IA is time consuming since all cells need to be examined by the operator and this may lead to significant subjective bias. For paediatric AM, the ideal technique should be automated, generate FC-type data, use very small numbers of cells and be able to process stored fixed cells. Laser scanning cytometry (LSC) is a new technique that potentially fulfils these requirements by combining the benefits of FC with those of IA and is built around the epi-fluorescence microscope [10]. Cells are first immobilised on a conventional glass slide by centrifugation or adherence, and after immunostaining are scanned sequentially by two lasers (argon and helium-neon, emitting at 488 and $633 \mathrm{~nm}$, respectively). The software recognises clusters of fluorescent pixels (pixelated clusters), these clusters being the primary "triggering" parameter for each cell. The 
process whereby the software identifies pixelated clusters in individual cells is called "cell capture". To date, the most common method of capturing cells is for the software to identify nuclei stained with a fluorescent dye [11-13]. To obtain immune receptor data from the cell surface and cytoplasm after nuclear capture, a "contour" is generated at a fixed distance from the nuclear perimeter. Fluorescent data are then generated within this contour. An alternative method of cell capture is to use the "intrinsic" properties of cells such as forward scatter [14] or autofluorescence. In these circumstances the software draws a contour around the entire cell, and the cytoplasm is assumed to be anything within this contour. An additional peripheral contour is therefore not necessary.

LSC has successfully been used to examine the phenotype of many cell types including blood leukocytes [15] and eosinophils and bronchial epithelial cells in sputum [11], but has not been used to study the AM immune phenotype. LSC may be ideally suited to the study of paediatric AM. First, AM can be stored as cytospins and antigens identified using fluorescentlabelled monoclonal antibodies in batches. Second, the fluorescent intensity of AM can be automatically measured in a few minutes using as little as a 1,000 cells. Therefore, the applicability of LSC to the study of paediatric AM was assessed here. The criteria for a successful scan were defined as: 1) the accurate identification of the entire cytoplasm of individual AM; 2) the ability to compensate for groups of AM that are adherent to each other; and 3) the generation of fluorescence intensity data from the cytoplasm of individual AM. The standard FC-derived techniques of forward scatter and autofluorescence were used for identifying AM, followed by the standard LSC technique of nuclear capture after staining. In addition, a novel method of AM capture was used whereby individual AM were captured after staining with a pan-macrophage marker (CD68).

\section{Materials and methods}

\section{Study subjects}

Healthy children undergoing elective surgical procedures were recruited to this study. Children were excluded for any of the following reasons: congenital heart disease, ongoing chronic pulmonary pathology, preterm delivery requiring ventilation, or symptoms suggestive of a lower respiratory tract infection in the past 6 weeks. This study had approval from the ethics committee and written informed consent was obtained from the parents of all children participating in the study. Where appropriate verbal consent was also obtained from the child.

\section{Study design}

Three techniques were assessed for their ability to identify AM. First, the standard FC techniques for identifying AM, i.e. intrinsic forward scatter and autofluorescence. Second, the ability of the LSC to capture and contour around AM nuclei, stained with the nucleic acid dye propidium iodide (PI). Thirdly, capture of whole AM when the cytoplasm was stained with a fluorescently conjugated monoclonal antibody to CD68, which reacts selectively with a specific $110-\mathrm{kDa}$ cytoplasmic glycoprotein present in mononuclear phagocytes. Slides were processed as outlined below and where data was obtainable for any of the above techniques the "sensitivity" and "positive predictive value" of the technique was tested as well as the repeatability of the analysis.

\section{Methods}

Bronchoalveolar lavage. All children received a general anaesthetic. At induction, either an inhalational agent or propofol was used according to the preference of the anaesthetist. Immediately after intubation and within 5 min of induction of anaesthesia, children underwent nonbronchoscopic BAL as previously described [16]. Briefly, the child's head was turned to the left and a suction catheter (six French Gauge $(\mathrm{FG})<1$ yrs, eight FG 1-5 yrs and $10 \mathrm{FG}>5$ yrs) was wedged in the lower airway. A total $1 \mathrm{~mL} \cdot \mathrm{kg}$ body weight ${ }^{-1}$ (maxi- $^{-}$ mum $20 \mathrm{~mL}$ ) of sterile saline at room temperature was instilled and immediately aspirated into a suction trap using gentle negative pressure (100-200 $\mathrm{mmHg}$ ). The procedure was repeated twice. Pooled bronchoalveolar lavage fluid (BALF) was stored on ice prior to processing.

Processing of samples. BALF was immediately centrifuged at $250 \times g$ for $5 \mathrm{~min}$ at $4^{\circ} \mathrm{C}$. After removal of the supernatant the cell pellet was resuspended in phosphate-buffered saline (PBS) (Sigma-Aldrich Company, Dorset, UK). This procedure was repeated twice and the cells resuspended in PBS at a final concentration of $1 \times 10^{5}$ cells $\cdot \mathrm{mL}^{-1}$. A total $1 \times 10^{4}$ unfixed cells were cytocentrifuged at $800 \mathrm{rpm}$ for 5 min onto several glass microscope slides (Shandon Scientific, Runcorn, Cheshire, UK). Slides were then air-dried for $1 \mathrm{~h}$ before fixing in chloroform:acetone mix at a ratio of $1: 1$ for $10 \mathrm{~min}$. After fixation, slides were air dried for a further hour prior to storing for up to 18 months at $-20^{\circ} \mathrm{C}$ until immunoassayed. The BALF cell differential count was determined prior to fixation by staining a slide with Diff-Quik (Dade Behring, Deerfield, IL, USA) and counting $>300$ leukocytes. Slides were brought up to room temperature and washed in PBS for $10 \mathrm{~min}$. Slides were processed for each child in one of three ways.

Capture using autofluorescence and forward scatter. Slides were left unstained in order to assess contouring around forward scatter and autofluorescent properties of the cell.

Capture using nuclear staining. To one slide, PI (SigmaAldrich) was added at a concentration of $20 \mu \mathrm{g} \cdot \mathrm{mL}^{-1}$ with Ribonuclease H (Sigma-Aldrich) at a concentration of $15 \mu \mathrm{g} \cdot \mathrm{mL}^{-1}$ for $30 \mathrm{~min}$ at room temperature 
in the dark. Preliminary work using concentrations of PI ranging from $20-100 \mu \mathrm{g} \cdot \mathrm{mL}^{-1}$ achieved the same intensity of staining.

Capture using a pan-macrophage monoclonal antibody. Two slides from each child were processed using standard immunostaining techniques after blocking with goat serum for $25 \mathrm{~min}$. One slide was incubated with a mouse monoclonal antibody to human CD68 (BD Pharmingen, San Diego, CA, USA) in goat serum at a final concentration of $10 \mu \mathrm{g} \cdot \mathrm{mL}^{-1}$, and one with an appropriate isotypic control (mouse immunoglobulin (Ig)G2b) (Serotec Ltd, Oxford, UK) in goat serum for $1 \mathrm{~h}$. Both slides were then washed twice in PBS before incubation with the secondary antibody Alexa Fluor 633 goat anti-mouse IgG2b (Molecular Probes, Leiden, the Netherlands) in goat serum at a final concentration of $13 \mu \mathrm{g} \cdot \mathrm{mL}^{-1}$ at room temperature for $30 \mathrm{~min}$ in the dark.

Laser scanning cytometry. All slides were mounted in Immunomount (Shandon Scientific) and processed on the LSC within $48 \mathrm{~h}$. Slides were scanned on the LSC (CompuCyte, Cambridge, MA, USA) and cells detected by contouring around one of the following: intrinsic cellular autofluorescence, forward scatter, nucleic acid PI and cytoplasmic CD68. For each slide at the start of scanning the threshold photomultiplier tube (PMT) sensitivity was adjusted such that the maximum detection of stained areas was achieved with a minimum detection of background staining. Once adjusted, the slide was scanned and data collected. A minimum of 1,000 cells per slide were analysed by the LSC using the $\times 20$ objective.

During scanning, images of the area being scanned were randomly selected and displayed on the data display window and inspected visually. This window shows pixelated areas and the presence of softwaregenerated contours. This enables an assessment of whether contours have appropriately identified single $\mathrm{AM}$, or have inappropriately characterised debris or clumps of cells as individual AM with a single contour, or even failed to detect and hence contour around AM. For each child a minimum of three data display windows were examined. AM were identified by the operator according to either the nuclear or cell size and contour shape and the following parameters were calculated: 1) the proportion of pixelated clusters that were morphologically single AM and were contoured around (sensitivity), and 2) the proportion of contours associated with pixelated clusters that were morphologically single AM (positive predictive value).

For AM stained with CD68, a qualitative assessment of specificity and negative predictive value for the sample was made by comparing the amount of pixelated clusters contoured around on the isotypic control slide compared to the CD68 stained slide. The variation in repeated measurements on the same subject (repeatability) was determined in AM from three randomly selected children. Two sets of slides obtained from a single sampling procedure and stored in an identical manner were immunostained with CD68 on two separate occasions and then scanned on the same LSC by the same observer each within $48 \mathrm{~h}$ of immunostaining.

After imaging, a presumed population of single AM were selected according to homogeneity and contour area from the software produced graphs of contour area versus pixel intensity. These "cells" were then displayed using the "relocation" feature on the software. For each sample, once scanning was recommenced, the first 36 contoured pixelated clusters that were within the selected area were visualised and the positive predictive value of the test for selecting single AM was calculated. This determined whether selecting a homogeneous population of cells with similar contour size would exclude debris or clumps of cells that had been inappropriately contoured around and increase the positive predictive value of the test for selecting single cells.

To determine whether it was possible to obtain fluorescent data within the contours, the integrated fluorescence was determined for each of the fluorochromes used. The integrated fluorescence for the active sensor is the background-corrected sum of the pixel values within the data contour (equivalent to FC-integrated fluorescence). The integrated fluorescence was corrected for contour area.

\section{Confocal microscopy}

To independently assess the specificity of CD68 fluorescent staining of AM and the degree of background staining, CD68-immunostained AM were imaged using the laser scanning confocal microscope (Bio-Rad Radiance 2000, Hertfordshire, UK). Positively stained slides were compared to the isotypic control and a qualitative assessment of the specificity and negative predictive value of CD68 immunostaining was estimated.

\section{Statistics}

The data are presented as medians and interquartile ranges (IQR). Sensitivities and positive predictive values were calculated according to the methods by Altman and Bland [17, 18]. In order to assess the repeatability of the test, the Bland-Altman repeatability coefficient was calculated. This defines the range in which $95 \%$ of measurements for sensitivity and positive predictive value would be expected to fall if the test was repeated on the same subject under the same conditions of measurement and is the probability of technical error. It was calculated by plotting the difference between the test and retest results for the three children studied and then calculating the mean and SD of the differences. The repeatability coefficient was calculated as twice the SD of the difference of the repeated measures [19].

\section{Results}

Twenty-eight children were studied (11 females) with an age range $0.1-14.1$ yrs. The baseline BALF 
cell data for these children are given in table 1. Detection of AM using their forward scatter properties was not possible and no data were obtained using this technique. Using AM autofluorescence, the LSC software contoured around some cells, but fluorescence was weak and variable between cells. Thus, many AM were either not detected at all or contoured around in clumps (fig. 1).

Capture after nuclear staining by PI did select nucleated cells. However, perimeter contours frequently overlapped into neighbouring cells because of the many eccentrically placed nuclei. In addition, multiple contours were frequently produced for each cell, as a result of double nuclear profiles (fig. 2). Therefore, these techniques did not meet the criteria for a successful scan and no further data were sought.

AM were successfully contoured around using CD68. The primary antibody was specific for the CD68 antigen on AM, as shown by the lack of pixelated clusters on slides stained with an appropriate isotypic control (fig. 3). Specific staining was confirmed by confocal microscopy which showed staining of cells that were morphologically AM (fig. 4). Contouring around the CD68-stained AM by LSC was complete with an occasional indentation due to reduced staining at the location of the nucleus (fig. 5).

Most cells were contoured around individually but a few were clumped and hence contoured around as clumps. The positive predictive value for detecting

Table 1. - Bronchoalveolar lavage fluid (BALF) parameters

\begin{tabular}{lcc}
\hline & Median & Interquartile range \\
\hline Age yrs & 1.5 & $0.4-5.6$ \\
BALF return \% & 54 & $37-63$ \\
Total cell numbers $1 \times 10^{6}$ & 1.4 & $0.4-2.0$ \\
Total AM count $1 \times 10^{6}$ & 1.2 & $0.4-1.9$ \\
\hline
\end{tabular}

AM: alveolar macrophage.

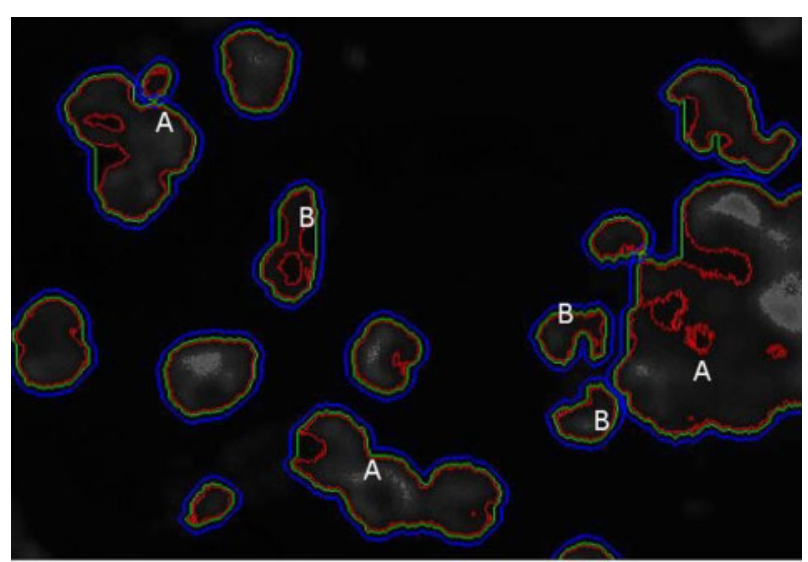

Fig. 1.-Autofuorescence as the contouring parameter. Alveolar macrophages were detected according to their autofluorescent properties and contoured around. This figure represents pixelated laser scan images produced by the software of the laser scanning cytometer, with contours around clusters of fluorescence. Fluorescent staining was both weak and variable resulting in either detection of clumps of cells $(\mathrm{A})$ or incomplete contouring of single cells (B).
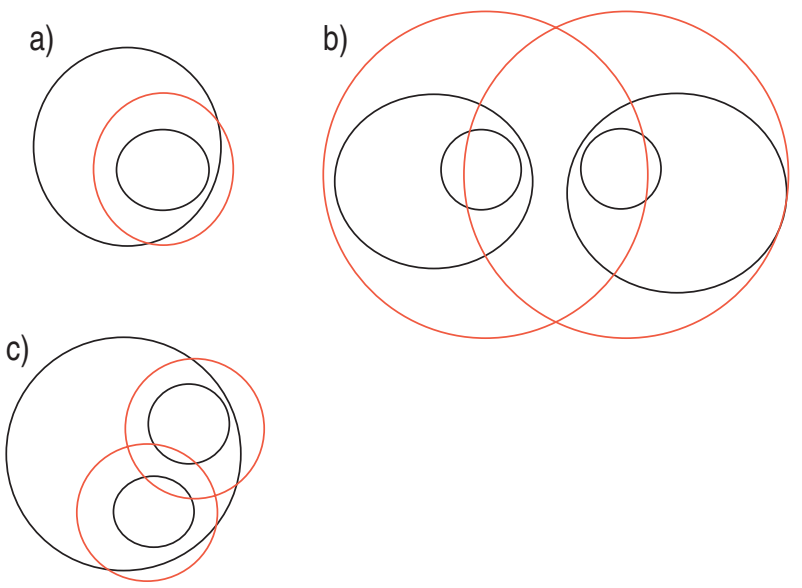

Fig. 2.-Propidium iodide as the contouring parameter. A diagrammatic representation of how the laser scanning cytometer (LSC) contours around alveolar macrophage (AM) nuclei. AM are detected by the LSC following nuclear staining with propidium iodide and the nucleus is contoured around (red oval). However, a) AM nuclei are not central within the cell and the software contours drawn around the nucleus do not capture the entire cell, unless b) the contouring parameters are increased at the expense of contours overlapping into neighbouring cells. c) AM on cytospins frequently have double or triple nuclear profiles resulting in multiple overlapping contours for individual cells.

single CD68-stained AM was 0.88 (IQR 0.79-0.99) and the sensitivity was 0.97 (IQR $0.92-1.00$ ). The repeatability coefficient for positive predictive value was 0.22 , and for sensitivity was 0.05 .

A population of CD68-stained AM was selected as probably being single by their contour size and homogeneity (fig. 6a). This selected population was rescanned, and using the relocation feature on the LSC, images were generated in order to confirm whether the software had captured single AM, debris or clumps of cells (fig. 6b). By selecting out a population of cells according to contour size, the positive predictive value for detecting single cells increased from 0.88 in the original scanned population to 0.94 (IQR 0.92-0.97).

Since the CD68 monoclonal antibody was bound to the CD68 antigen it could be used as an example of staining for an immune receptor, as well as a contouring feature. FC-type data was generated for all children and corrected for contour area. The median integrated fluorescence for CD68 immunostained AM was 7,725 (IQR 6,760-9,635) arbitrary units.

\section{Discussion}

This study reports, for the first time, a way of studying stored paediatric AM using the LSC. Contouring around the macrophage-specific cellular antigen CD68 is a sensitive and specific technique for detecting AM, with excellent test retest repeatability for sensitivity. The advantages of this technique are three-fold. First, the AM is completely contoured around allowing specific cellular or surface antigens to be further studied without the need for a software generated peripheral contour, as the authors have demonstrated with CD68. Second, AM can be easily discriminated from other cell 

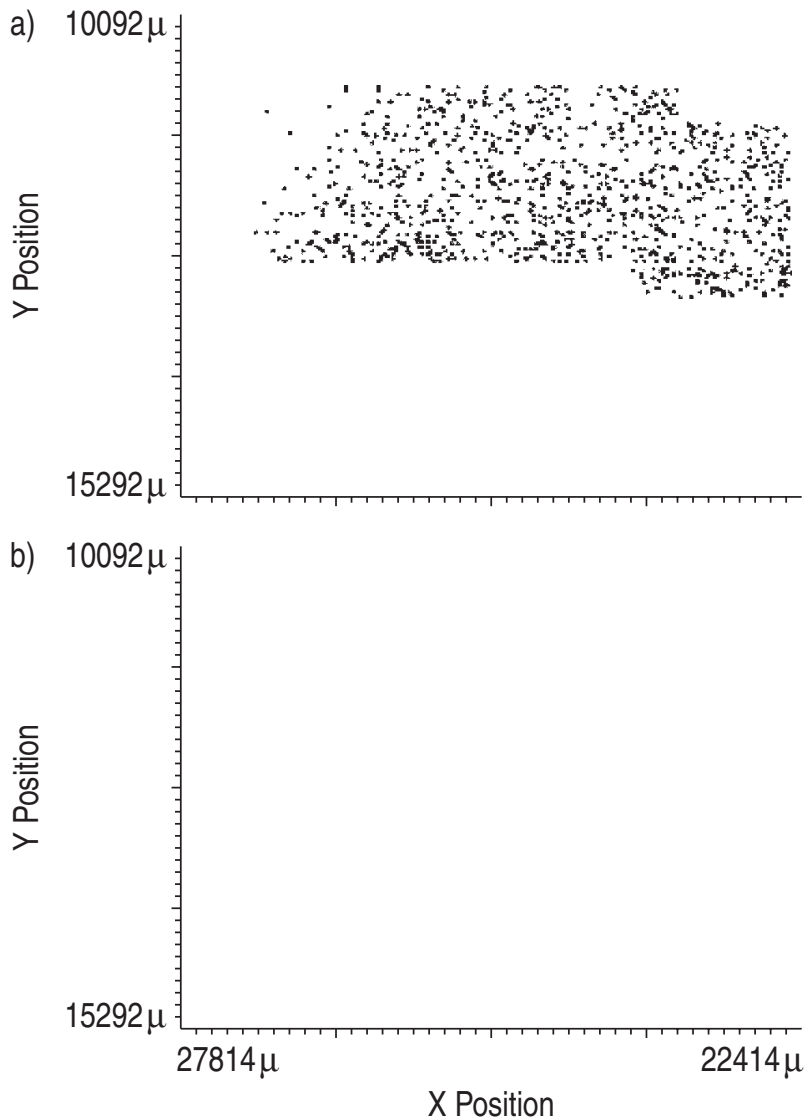

Fig. 3.-Specificity of CD68 immunostaining. Slides from the same patient were either immunostained with a) the primary antibody CD68 or b) an appropriate isotypic control. Each dot represents a pixelated cluster that has been contoured around and the $\mathrm{x} / \mathrm{y}$-axis equates to its position on the slide. As demonstrated by the absence of detectable pixelated clusters on the isotypic control slide the primary antibody is specific for CD68 on alveolar macrophages.

populations within the BALF sample. Third, most cells are contoured around singly; those that are contoured around as clumps can be easily excluded according to contour size.

The two previously reported methods for detecting cells using the LSC involve either staining the nucleus with a nucleic acid dye [11-13] or contouring according to scatter properties [14]. Nuclear contouring has been successfully employed for cells with single nuclear profiles and can discriminate different cell populations in peripheral blood according to the nuclear size and density of chromatin condensation [12]. The nucleus of the AM however is a less attractive cell localising and contouring parameter since AM are large cells (10-20 $\mu \mathrm{m}$ diameter) [20] with large eccentrically placed nuclei that are often horseshoe shaped and frequently appear bi- or even multi-nucleated when flattened on cytospins [21]. When the nucleus is used as the triggering parameter, the software often identifies binucleated cells as two separate AM. Furthermore, AM have a high propensity to homotypically aggregate [22] and contours from neighbouring nuclei frequently overlap (fig. 2). An additional problem with nuclear staining is that the chromatin

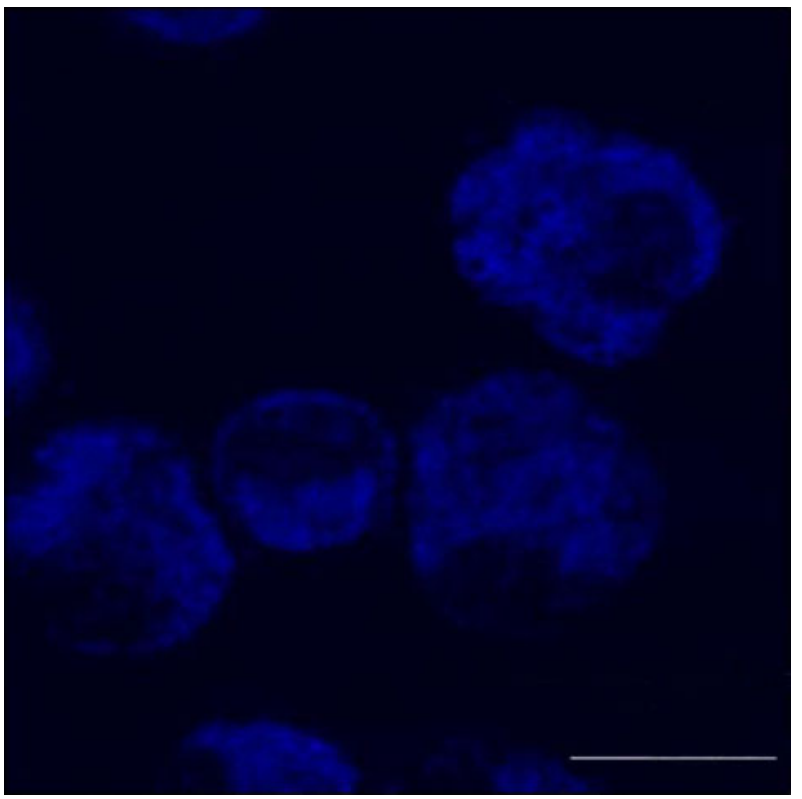

Fig. 4.-Confocal microscopy of alveolar macrophages (AM) immunostained with CD68 and Alexa 633. Slides were examined for specific AM staining using the confocal microscope. Cells which are morphologically AM can be seen in blue with no evidence of background staining. The eccentrically placed nuclei can be clearly seen as areas of reduced intensity stain, with uniform staining throughout the remaining cytoplasm. Internal scale bar $=10 \mu \mathrm{m}$.

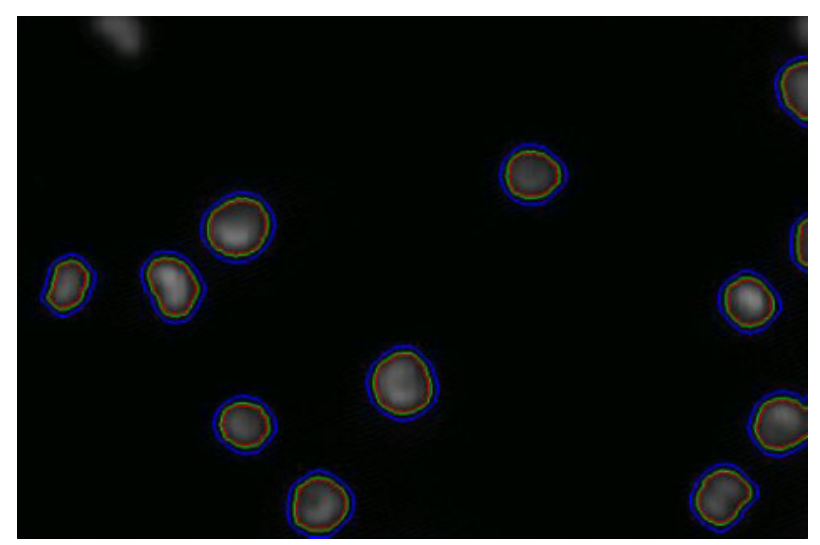

Fig. 5.-Contouring around CD68 immunostained alveolar macrophages (AM). AM have been immunostained with CD68. This figure represents pixelated laser scan images produced by the software of the laser scanning cytometer, with contours around clusters of fluorescence. The cell is contoured around completely with an occasional indentation at the site of the nucleus where the stain is less intense, as can be seen using confocal microscopy (fig. 4).

within the nucleus is not densely packed [23] and its density is dependent on cell age and nuclear size; hence, staining with PI is weaker at standard concentrations and contouring less consistent. As a result of the above problems, the authors' attempts to use the AM nucleus as the contouring parameter were not successful.

Adult AM (especially those obtained from smokers) are intensely autofluorescent and this, combined with 


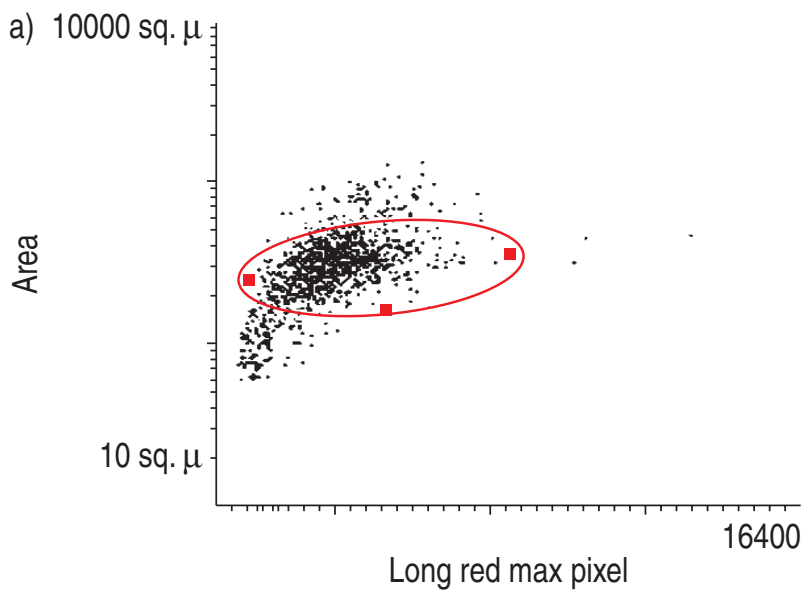

b)

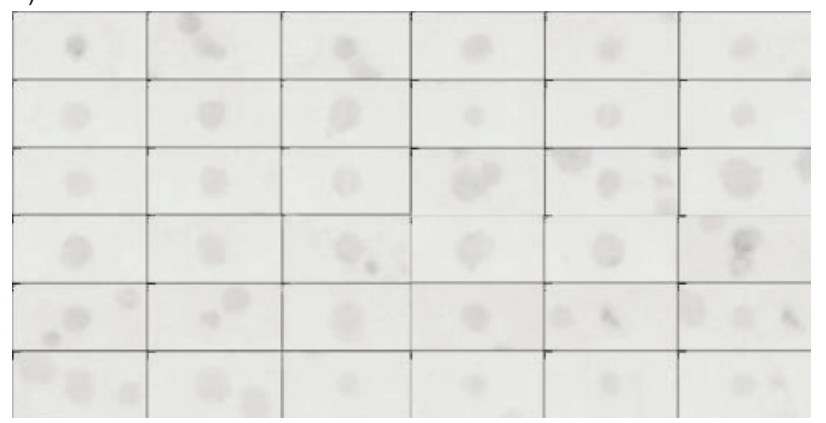

Fig. 6.-Selecting a representative population of contours presumed as being alveolar macrophages. a) Each dot represents a pixelated cluster contoured around by the laser scanning cytometer. The $\mathrm{x}$-axis represents the maximum fluorescence within the contour and the y-axis represents the contour area. A homogenous population of cells was selected (red ring) thus increasing the chance of eliminating debris (clusters smaller than the selected area) and cell clumps (clusters larger than the selected area). b) The selected population was then relocated, rescannned and the pixelated images visualised as seen in an example from one child. Here most images identify single cells.

their intrinsic forward scatter properties, have allowed them to be easily identified by FC [7]. AM sampled from young rats are much less autofluorescent than those sampled from older animals [24] and this may explain the weak autofluorescent properties of the paediatric samples. In addition, the process of storing cells first by applying centrifugal force, then fixing and freezing cells undoubtedly causes leaching of natural fluorochromes from cells [25], as well as altering AM morphology [26] and thus their forward scatter properties. For these reasons AM cannot be easily identified by these "intrinsic" properties. Light scatter has successfully been used as the LSC contouring parameter on live lymphocyte preparations [14] adherent to the slide, and the possibility for exploiting the forward scatter and autofluorescent properties of live AM requires further study before these can be excluded as useful contouring parameters.

What are the limitations of the present study? First, the accepted gold standard for determining the presence and intensity of cellular and surface antigens is currently FC. Ideally any new technique should be compared against this gold standard. Unfortunately, a direct comparison with $\mathrm{FC}$ was not made as many of the samples, especially from infants, contained insufficient cells to process them using both techniques. Second, although LSC and confocal microscopy confirm the absence of background staining and hence no contouring around cells which were not AM, it is possible that some AM were CD68 negative and not detected. Reports suggest that this accounts for $\sim 3 \%$ of AM [6].

To obtain data on other immune receptors, dual staining with CD68 and the receptor of interest will be necessary. One potential disadvantage of LSC, and indeed FC when dual staining, is the problem of "spillover" of one fluorochrome into a neighbouring PMT that is being used to detect a different fluorochrome (i.e. "spectral overlap"). For a fluorochrome to be a useful contouring parameter there has to be minimal spill-over into neighbouring PMT so that the presence and intensity of the antigens under test can be studied. In order to avoid spectral overlap the authors suggest using a fluorochrome such as Alexa Fluor 633 as the contouring parameter since it is excited by the $\mathrm{HeNe}$ laser at $633 \mathrm{~nm}$ and emits at a wavelength of $670 \mathrm{~nm}$ far beyond the detection parameters of the other PMTs. These dyes also have the benefits of low fluorescence quenching upon binding to proteins and hence can be stored for up to $48 \mathrm{~h}$ with minimal bleaching. However, one disadvantage of Alexa 633 is that it is not visible to the naked eye and hence an assessment of staining using standard immunofluorescence is not possible. Therefore, the authors also immunostained some slides with R-phycoerythrin, which is excited by the argon laser and detected by the orange PMT and can be viewed with the naked eye. This detects fluorescent spectra close to that of the green and far-red PMT. The degree of compensation required for spill-over into these neighbouring PMT (compensation factor) was tested. For orange spill-over into green the compensation factor was $0.03 \%$ and for orange into far red it was $0.02 \%$ (data not shown) thus making this an acceptable contouring fluorochrome.

To conclude, laser scanning cytometry is suited to the study of paediatric alveolar macrophages since it uses very small numbers of cells ( $\sim 5,000$ per slide) and can be applied to stored slides. By using a pan-macrophage marker as the triggering parameter the authors were able to overcome the limitations of laser scanning cytometry and could both accurately identify alveolar macrophages and obtain fluorescence data from these cells. It is speculated that laser scanning cytometry will be an important methodology in the study of developmental changes in the immune profile of the human alveolar macrophages.

Acknowledgements. The authors would like to thank AstraZeneca Research and Development Discovery Bioscience (Loughborough, Charnwood, UK) for use of their laser scanning cytometer and the confocal microscope.

\section{References}

1. De Blic J, Midulla F, Barbato A, Clement A, Dab I, Eber E. Bronchoalveolar lavage in children. ERS Task Force on bronchoalveolar lavage in children. 
European Respiratory Society. Eur Respir J 2000; 15: 217-231.

2. Bunn HJ, Hewitt CR, Grigg J. Suppression of autologous peripheral blood mononuclear cell proliferation by alveolar macrophages from young infants. Clin Exp Immunol 2002; 128: 313-317.

3. Fleit HB, Weiss RA, Chanana AD, Joel DD. Fc receptor function on sheep alveolar macrophages. J Leukoc Biol 1986; 40: 419-431.

4. Kradin RL, McCarthy KM, Schneeberger EE. Opsonic receptor function is reduced on the surface of newborn alveolar macrophages. Am Rev Respir Dis 1986; 133: 238-244.

5. Kradin RL, McCarthy KM, Preffer FI, Schneeberger EE. Flow-cytometric and ultrastructural analysis of alveolar macrophage maturation. J Leukoc Biol 1986; 40: 407-417.

6. Umino T, Skold CM, Pirruccello SJ, Spurzem JR, Rennard SI. Two-colour flow-cytometric analysis of pulmonary alveolar macrophages from smokers. Eur Respir J 1999; 13: 894-899.

7. Maus U, Rosseau S, Seeger W, Lohmeyer J. Separation of human alveolar macrophages by flow cytometry. Am J Physiol 1997; 272: L566-L571.

8. Lemjabbar H, Gosset P, Lechapt-Zalcman E, FrancoMontoya ML, Wallaert B, Harf A. Overexpression of alveolar macrophage gelatinase B (MMP-9) in patients with idiopathic pulmonary fibrosis: effects of steroid and immunosuppressive treatment. Am $J$ Respir Cell Mol Biol 1999; 20: 903-913.

9. Allen JN, Moore SA, Liao Z, Wewers MD. Changes in mononuclear phagocyte microtubules after endotoxin stimulation. I. Changes in microtubule stability. Am J Respir Cell Mol Biol 1997; 16: 119-126.

10. Martin-Reay DG, Kamentsky LA, Weinberg DS, Hollister KA, Cibas ES. Evaluation of a new slidebased laser scanning cytometer for DNA analysis of tumors. Comparison with flow cytometry and image analysis. Am J Clin Pathol 1994; 102: 432-438.

11. Woltmann G, Ward RJ, Symon FA, Rew DA, Pavord ID, Wardlaw AJ. Objective quantitative analysis of eosinophils and bronchial epithelial cells in induced sputum by laser scanning cytometry. Thorax 1999; 54 : 124-130.

12. Bedner E, Burfeind P, Gorczyca W, Melamed MR, Darzynkiewicz Z. Laser scanning cytometry distinguishes lymphocytes, monocytes, and granulocytes by differences in their chromatin structure. Cytometry 1997; 29: 191-196.
13. Gerstner A, Laffers W, Bootz F, Tarnok A. Immunophenotyping of peripheral blood leukocytes by laser scanning cytometry. J Immunol Methods 2000; 246: 175-185.

14. Clatch RJ, Foreman JR, Walloch JL. Simplified immunophenotypic analysis by laser scanning cytometry. Cytometry 1998; 34: 3-16.

15. Clatch RJ, Foreman JR. Five-color immunophenotyping plus DNA content analysis by laser scanning cytometry. Cytometry 1998; 34: 36-38.

16. Heaney LG, Stevenson EC, Turner G, Cadden IS, Taylor R, Shields MD. Investigating paediatric airways by non-bronchoscopic lavage: normal cellular data. Clin Exp Allergy 1996; 26: 799-806.

17. Altman DG, Bland JM. Diagnostic tests 2: Predictive values. BMJ 1994; 309: 102.

18. Altman DG, Bland JM. Diagnostic tests. 1: Sensitivity and specificity. BMJ 1994; 308: 1552.

19. Bland JM, Altman DG. Statistical methods for assessing agreement between two methods of clinical measurement. Lancet 1986; 1: 307-310.

20. Fathi M, Johansson A, Lundborg M, Orre L, Skold CM, Camner P. Functional and morphological differences between human alveolar and interstitial macrophages. Exp Mol Pathol 2001; 70: 77-82.

21. Appel J, Szule P. Electron microscopy of macrophages obtained from bronchial lavage-fluid. Acta Morphol Hung 1986; 34: 163-170.

22. Grigg J, Kukielka GL, Berens KL, Dreyer WJ, Entman ML, Smith CW. Induction of intercellular adhesion molecule-1 by lipopolysaccharide in canine alveolar macrophages. Am J Respir Cell Mol Biol 1994; 11: 304-311.

23. Kawahara K, Itoh S, Honjou S, Tagawa Y, Akamine $\mathrm{S}$, Takahashi $\mathrm{T}$. The RNA content of alveolar macrophages in canine lung allografts. J Surg Res 1993; 55: 131-134.

24. Bakker JM, Broug-Holub E, Kroes H, van Rees EP, Kraal G, van Iwaarden JF. Functional immaturity of rat alveolar macrophages during postnatal development. Immunology 1998; 94: 304-309.

25. Laing RA, Fischbarg J, Chance B. Noninvasive measurements of pyridine nucleotide fluorescence from the cornea. Invest Ophthalmol Vis Sci 1980; 19: 96-102.

26. Van Meir F. Planimetry of bronchoalveolar macrophages. Importance of preparation and staining techniques. Anal Quant Cytol Histol 1991; 13: 261268. 